\title{
Controlling Shear Stress in a Suspension Culture using Couette Flow for Efficient Proliferation of HEK 293 Cells
}

\author{
Nur Khatijah Mohd Zin ${ }^{1,2}$, Katsuhisa Sakaguchi ${ }^{1,2 *}$, Yuji Haraguchi ${ }^{2}$, Azuma Takahashi ${ }^{1}$, Sara Suzuki ${ }^{1}$, Takanobu Yagi ${ }^{1}$, Tatsuya Shimizu ${ }^{2}$ and Mitsuo Umezu ${ }^{1}$ \\ ${ }^{1}$ School of Advance Science and Engineering, TWIns, Waseda University, Wakamatsu-cho, Shinjuku-ku, Tokyo, Japan \\ ${ }^{2}$ Institute of Advanced Biomedical Engineering and Science, TWIns, Tokyo Women's Medical University, Tokyo, Japan
}

"Corresponding author: Sakaguchi K, School of Advance Science and Engineering, TWIns, Waseda University, 2-2 Wakamatsu-cho, Shinjuku-ku, Tokyo 162-8480, Japan, Tel: +81-3-5367-9945; Fax: +81-3-3359-6046; E-mail: katsuhisa@toki.waseda.jp

Received date: May 12, 2016; Accepted date: May 31, 2016; Published date: June 06, 2016

Copyright: @ 2016 Mohd Zin NK, et al. This is an open-access article distributed under the terms of the Creative Commons Attribution License, which permits unrestricted use, distribution, and reproduction in any medium, provided the original author and source are credited.

\begin{abstract}
The suspension culture system is an increasingly popular method of culturing cells not only because of its up scaling ability, but also the non-enzymatic procurement of cells that is crucial for biomedical research, especially in the fields of pharmacology and regenerative medicine. Hypothetically, by controlling and reducing the shear stress applied to cells in a culture system, the higher viability and proliferation rates. In this study, we analyzed HEK 293 cells cultured with a commercially available spinner flask and our newly developed spinner flask which utilizes the theory of Couette flow for controlling shear stress. Fluid analysis and metabolic analysis of the cultured cells were measured at three different rotational speeds, 40, 50 and $60 \mathrm{rpm}$. It was apparent that 50 rpm was by far the best speed to proliferate the cells. A further viability test was also done in order to validate our hypothesis. Furthermore, by using the metabolic analysis results, it was observed that in the controlled stress system, the consumption of glucose doubled and lactate production was significantly higher compared to cells that were maintained in the conventional suspension method. Thus, Couette flow based suspension culture system will be a major contributor to the future biomedical and pharmacological field.
\end{abstract}

Keywords: Bioreactor; Couette Flow; HEK 293 Cells; Shear Stress; Suspension Cell Culture

\section{Introduction}

Biopharmaceutical products such as hormones, antibody preparations, blood factors, cytokines, growth factors and vaccines have become a major interest worldwide [1]. In 2013 alone, 94 billion dollars (USD) of biopharmaceutical products were sold in the US and the European Union. In 1982 the first product was approved by the USFDA, which was a recombinant human insulin (Humulin, Eli Lilly, Indianapolis, USA), a product of Escherichia coli (E-coli). Currently, mammalian cell culture has come to the forefront of biopharmaceutical production due to its capability of properly folding, assembly, and post-translational modification (PTM) of proteins [2]. The first ever genetically modified product was human tissue plasminogen activator (tPA, Activase, Genentech, CA, USA) that obtained market approval in 1986 [2]. Presently, mammalian cells including human cells are currently being used for over $50 \%$ of all biopharmaceuticals being produced [1]. Within the mammalian cells, including human embryonic kidney (HEK 293) and Chinese hamster ovary $(\mathrm{CHO})$ cells, are the most widely expressed cells due to the effectiveness and susceptibility of these cells to be transfected and their high proliferation rate. In addition to being able to survive the suspension culture system, these cells are regularly used for producing a diverse range of recombinant proteins [3-6]. As expected, industrially produced recombinant proteins from mammalian cells are also used in a wide variety of biomedical research at laboratories around the world.

In recent years, the advancement of suspension culture has opened diverse opportunities for ways of managing controllable variables [7]. Additionally, according to Ryu et al. [8] suspension culture has an advantage over monolayer culture on the basis of ease of scaling up, homogeneity condition as well as the growth area. Moreover, according to Zweigerdt et al. [9] compared to a conventional monolayer culture system, suspension culture saves space is less expensive cost, requires less manual labor. In addition to being a simple enzyme-free cell harvesting method it offers the possibility of a fully automated process. Current suspension culture technology has made it possible for moderate scale production of antibodies using stable cell lines that are required for functional and structural analysis [10]. Utilizing this method on a larger scale, a more extensive production of antibodies and probably proteins could be possible.

Recently, our laboratory reported a high-throughput electrophysiological analysis by using a 384-well patch-clamp system. This system is able to record up to 384 samples simultaneously [11]. Various inhibitory effects of the human ether-a-go-go-related gene (hERG) channel are able to be analyzed by the high-throughput patchclamp system on both HEK 293 and CHO cells expressing hERG gene. The patch-clamp system allows the detection of inhibitory effects from a diverse number of drugs against various ion channels in a highthroughput system within the random screening of various libraries [12]. This will also be a major contributor to the advancement of pharmaceutical drug testing and drug development. In the highthroughput random screening, an enormous number of cells are necessary. Thus, in the field of pharmacology and toxicology, an optimal suspension culture system could definitely be a key contributor in the fields of drug development, quality assurance, and quality control. Using this technology could exponentially accelerate the speed of testing and lower the cost $[13,14]$.

This being said, other cells such as human embryonic stem cells (hESC) [15] and induced pluripotent stem cells (hiPSC) [16,17] were 
also investigated. These kinds of tests are critically important in the regenerative medical and the pharmacological fields. Differentiated stem cell-derived cells such as cardiomyocytes and hepatocytes are vital in the field of pharmacology and toxicology; as a consequence, this would be a logical next step for regeneration and tissue engineering $[18,19]$. This is particularly important for drug testing in areas that pose life-threatening risks such as arrhythmia and cardiotoxicity. Due to its proliferation ability, hESCs and hiPSCs are being cultured using various suspension culture systems in various laboratories, including our own laboratory [9,20-24].

Though conventional monolayer cultures could achieve similar results, another critical difference that suspension culture offers is improved distribution of nutrients, growth factors both within and additional to the culture medium, as well as improved oxygen diffusion. Insufficient nutrients or oxygen could inhibit cell proliferation resulting in apoptosis. Due to the constant media movement, all cells are able to receive equal amounts of nutrients, oxygen and growth factors as well as dissipate secretions more evenly [25]. The suspension culture system also provides a significantly larger area for the cells to grow compared to a conventional monolayer culture system. This method not only produces a greater number of cells in within the same culturing time frame but according to our results also achieved similar cell viability. The ease of cell procurement without the need of enzyme treatment minimizes the need for cell treatment, which could be an advantage for future clinical applications.

Despite these encouraging results, there are still a number of challenges to implementing the suspension culture system. Challenges such as excessive stirring, uneven stress distribution; cell-to-cell and cell-to-wall collisions are just a few of the obstacles to be resolved. In order to utilize the suspension culture system, adequate flows are important to minimize the cell damage and to maximize cell viability. With this in mind, we decided to take on the challenge to incorporate mechanical agitation while controlling shear stress distribution. We were looking for a mechanism that could reduce the turbulence in a stirred suspension culture and provide a computable shear stress at most points. In this study, we developed a suspension culture device that was inspired by the theory of Couette flow, which is a laminar flow of a viscous fluid in the narrow gap between two parallel surfaces, one of the surface is moving relative to the other [26,27]. With this theory, the idea of controlling shear stress and utilizing a three-dimensional (3-D) printer, this idea was then tested using HEK 293 cells that were proliferated efficiently and had a higher viability than those from a conventional system.

\section{Materials and Methods}

\section{Cell culture}

Human Embryonic Kidney; HEK 293FT cells (Invitrogen Life Technologies, CA, USA) were maintained with a culture medium mix of Dulbecco's modified Eagle's medium (DMEM; Sigma-Aldrich, St. Louis, MO, USA) supplemented with $4 \mathrm{mM}$ L-glutamine (Invitrogen), $1 \mathrm{mM}$ sodium pyruvate (Invitrogen), $0.1 \mathrm{mM}$ non-essential amino acids (Invitrogen), and 10\% fetal bovine serum (FBS; Japan Bio Serum, Nagoya, Japan)]. Media were changed every 2 to 3 days for 4 to 5 days to reach 80 to 85 percent $(80-85 \%)$ confluence before harvested to be used in the suspension culture. Cells were cultured at $37^{\circ} \mathrm{C}$ in a humidified atmosphere of $5 \% \mathrm{CO}_{2}$.

\section{Suspension culture device}

Spinner flasks used for the experiment by incorporating the theory of a viscometer, a small cylinder was added to the flask (Figures 1a and 1b), to successfully produce a controlled shear stress bioreactor and was compared to the commercially available spinner flask which were purchased from Wheaton Inc. (Millville, NJ, USA), which is shown in (Figures $1 \mathrm{a}$ and $1 \mathrm{c}$ ). (Figures 1 (b-ii) and 1 (c-ii)) are illustrations to further clarify the inner part of the spinning flask. The cylinder was designed by using a computer-aided design system (Inventor) (Autodesk, San Rafael, CA, USA), and created with a rapid prototyping system (EDEN350TM) (Objet Geometries, Rehovot, Israel). The cylinder was made from a photopolymer (FullCure ${ }^{\circ} 720$ ) (Objet Geometries).

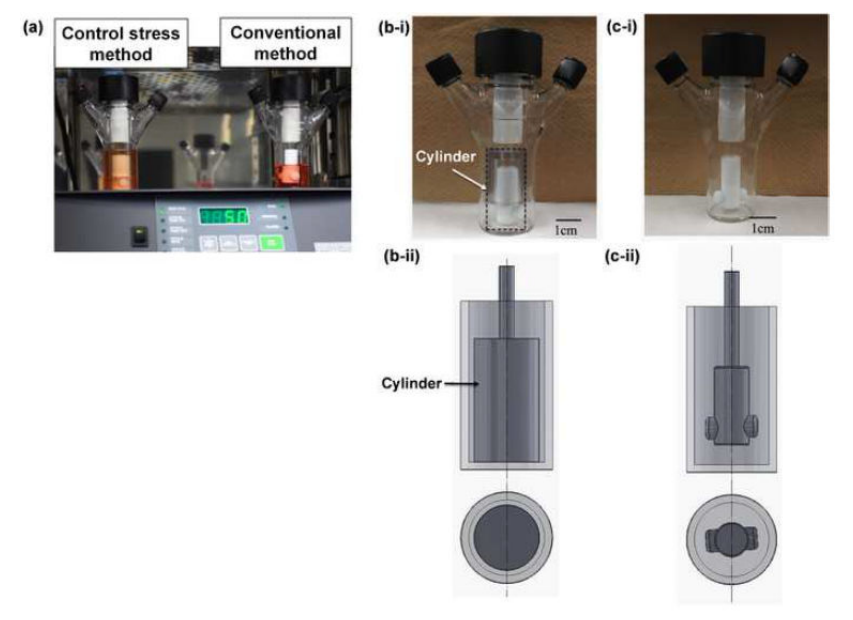

Figure 1: Suspension culture system used in this study. (a) The photograph on the left shows an over-view image of the suspension culture system set up in a $37^{\circ} \mathrm{C}$, humidified atmosphere of $5 \% \mathrm{CO}_{2}$ (left: the proposed device, right: a conventional device). (b-i) and (b-ii) are a photograph and schematic illustration of a controlled shear stress device, respectively. (c-i) and (c-ii) are a photograph and schematic illustration of a conventional spinner flask, respectively.

HEK 293FT cells (initial cell concentration: $2.5 \times 10^{6}$ cells $/ 20 \mathrm{~mL}$ ) in both suspension culture systems were cultured at $37^{\circ} \mathrm{C}$ in a humidified atmosphere of $5 \% \mathrm{CO} 2$. Both the conventional method and controlled stress method bioreactors were running concurrently for 4 consecutive days. Three different speeds of 40,50 and $60 \mathrm{rpm}$ were used and repeated 4 times each. Living cell numbers and cell viability were determined by the trypan blue-exclusion test.

\section{Fluid dynamical analysis}

Particle image velocimetry (PIV) was used to analyze the difference in fluid movement within the suspension culture system as shown in Figure 2a. This was done by lacing the measured fluid with fluorescent microspheres. The density of the particles was adjusted to perfectly match the density of the media, which has the same density as water. Velocity mapping was then acquired by using a stereoscopic PIV system (LA Vision Inc., MI, and USA) as described in a previous report [28]. 


\section{Metabolic Analysis}

The metabolic analysis of 3-D cultured HEK 293FT cells were conducted by measuring glucose consumption and lactate production in the culture media. The total glucose consumption and total lactate production in the culture media were measured over 4 days. The concentrations of glucose and lactate were determined by the method described previously [29-32].

\section{Statistical analysis}

All data are expressed as means \pm standard deviation. An unpaired Student t-test was performed to compare two groups. One-way analysis of variance was used for multiple group comparisons. If the Fdistribution was significant, a Tukey's test was used to specify differences between groups. A value of $\mathrm{p}<0.05$ was considered statistically significant.

\section{Results and Discussion}

\section{Fluid dynamical analysis}

The fluid movement in both the conventional and controlled shear stress methods of the suspension system was recorded and analyzed as shown in Figures 2a, 2b and 2c. In controlled shear stress method, design of the flow follows similar fluid movement to a viscometer, in which theory of Couette flow was employed. Both shear stress and vorticity in the conventional method was considerably higher than the controlled stress method. However, if the design is considerably shrunk, this theory may not be applicable due to the difference it could make to the fluid flow further to the spinner. As a result, a smaller clearance in the spinner flask was used. Hence, results utilizing the controlled stress method are graphically depicted in Figures $2 \mathrm{~d}$ and 2e. From the figure, it is clear that both shear strength and vorticity at all rotational speeds were significantly higher in the conventional method compared to the controlled stress method. Moreover, shear strength at $50 \mathrm{rpm}$ for the controlled stress method was lower than that found in the conventional method at $40 \mathrm{rpm}$. Also, it was observed that the vorticity recorded at $60 \mathrm{rpm}$ in the controlled stress method was lower than the vorticity recorded at $50 \mathrm{rpm}$ in the conventional method. On the other hand, a drastic increase in shear strength could be seen at 60 rpm in both systems.

\section{Suspension culture of HEK 293FT cells}

It was hypothesized that if the proposed device could suppress the shear stress and vorticity, it would induce better culture conditions in a suspension cell culture system. Thus, we prepared a suspension culture of HEK 293FT cells using both the conventional method and the controlled shear stress method. In the conventional system, after 4days cultivation at $50 \mathrm{rpm}$ the total cell numbers were highest with a cultivation of $1.3 \times 107$ cells. However, the number of cells increased approximately twofold to $2.1 \times 10^{7}$ cells using the controlled stress method at the same rotational speed (Figure $3 \mathrm{a}$ ), which was 8.4 times more than the number of seeded cells.

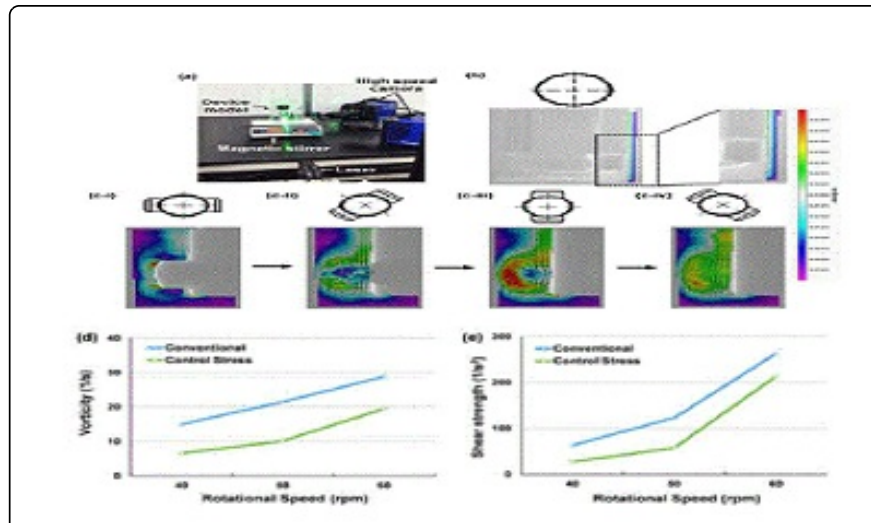

Figure 2: Fluid dynamic analysis using a particle image velocimetry device. Particle image velocimetry (PIV) device set up using a model of the spinner flask used for the suspension culture experiments (a). Schematic of the controlled shear stress method results (b).Schematic of the conventional method result at different phases (i) $0^{\circ}$ (ii) $45^{\circ}$ (iii) $90^{\circ}$ (iv) $135^{\circ}$ (c). Graphical depiction of the vorticity results (d). Graphical depiction of the shear strength results (e).

This result shows that there was more metabolic reaction in the proposed method as opposed to the conventional method. This shows that the rotation speed was optimal in the suspension culture system and by using the proposed device, the total cell numbers tended to be higher at all rotation speeds compared with the conventional system (Figure 3a). More importantly, the viability of proliferated cells in the proposed system tended to increase at all rotational speed (Figure $3 b$ ).

The best result was procured from the controlled stress method at $50 \mathrm{rpm}$, which recorded $71 \%$ living cells, compared to the $58 \%$ living cells recorded from the conventional method at the same speed. The worst result, however, was slightly less than $45 \%$, which was recorded in the conventional method at $60 \mathrm{rpm}$.

On the other hand at the same speed, the controlled stress method produced 58\% living cells, which is on par with the result procured for the conventional method at $50 \mathrm{rpm}$. It was also discovered that at 50 rpm the results were significant different between the two systems. Next, the condition of the cells was also evaluated by using cell metabolic analyses, glucose consumption and lactate production at 50 rpm where the result has the most significance.

The glucose consumption and lactate production of proliferated cells using the proposed method were significantly higher than those using the conventional method (Figure 3c). These results confirmed that the proposed method allowed us to efficiently proliferate cells while suppressing cell damage. Couette flow, which is a basic theory in fluid mechanics, is the laminar flow of a viscous fluid in the narrow space between two parallel surfaces, one of that is moving relative to the other $[25,26]$. In this study, we developed a suspension culture device, which was inspired by the theory of Couette flow, and showed that HEK 293FT cells were proliferated efficiently, while depressing cell damage compared with a conventional suspension culture device.

In the proposed suspension culture device, shear stress and vorticity were decreased compared with the conventional system. It is interesting that by covering a small portion of the stirrer, a significant reduction in shear stress and vorticity could be seen. 


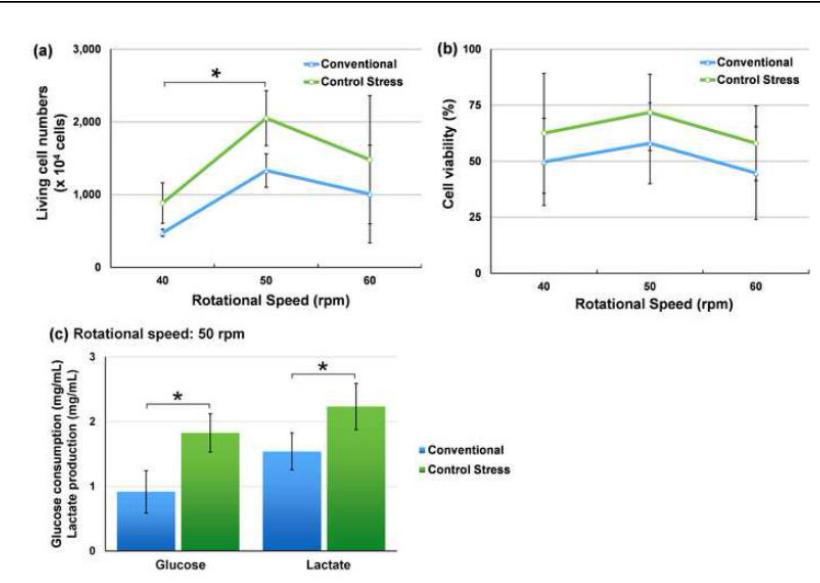

Figure 3: Living cell numbers, cell viability and metabolic analysis in the suspension culture of HEK 293FT cells. Quantification data of living cell numbers (a). Cell viability (b). Graphical depiction of glucose consumption and lactate production (c). The data are expressed as mean \pm standard deviation $(n=4)$.

From the results obtained, it was shown that the controlled stress method suspension culture system has better distributed shear stress; resulting in a greater proliferation of cells. However, the conventional version had more turbulence resulting in non-uniform stress, making proliferation more difficult and consequently caused more cell death.

The highest result obtained in this study was $71 \%$, which was by the controlled stress method at $50 \mathrm{rpm}$, although this might not be the best result possible in a suspension culture system, and further tests will be required. Due to the constraint of media volume at $20 \mathrm{~mL}$ and the high concentration of cells $\left(2.1 \times 10^{7}\right.$ living cells $)$ in the spinner flask, the lack of nutrients on the third and fourth day might be a reason for the apoptosis of the cells. Possible solutions to overcome this issue could include using the same concentration of cells in a bigger flask or by changing the media every other day rather than once every four days. Bearing these issues in mind, it is thought that it would be possible scale up the culture system to proliferate more cells at a quicker pace. This would then meet the needs of medical transplantation, which require at least $1 \times 10^{9}$ cells for one patient [9].

In this report, HEK 293 cells were efficiently proliferated in the proposed suspension culture system. At present, the system is being applied to the proliferation of other cells, including $\mathrm{CHO}$ cells expressing the human immunoglobulin $G$ (IgG) gene for producing antibodies [33], HEK 293 cells expressing the human ion channel gene for high-throughput patch-clamp analysis, and hiPSCs for the preclinical research in regenerative medicine and pharmaceutical drug testing. Promising preliminary result has been found for HEK-hERG and iPS cells using this system, but yet to be published. Investigations into various issues such as the detection of inhibitory effects from a diverse number of drugs against various ion channels could be done on a larger scale and at quicker speed when more cells can be procured. Therefore, we are confident that the proposed suspension culture system can be used as a powerful tool in various fields of biomedical research and pharmaceutical science.

Using this system on a larger scale would hopefully increase the production of cells as well as the production of biopharmaceutical products within a shorter time frame. Since this culture system does not require a very large space in the laboratory, it is hoped that it would also help with the culturing of the large number of cells needed for transplantation.

In conclusion, our hypothesis based on the theory of fluid mechanics, it was confirmed that our developed device did suppress shear stress and vorticity, which induced better culture conditions in a suspension cell culture system. These results will be of value in future of biomedical research, especially in the development of biomedical technology and pharmaceutical science. With this, it is presumable that fluid mechanics would be a bridge to integrate between mechanical engineering, medical and pharmacological field.

\section{Acknowledgement}

We would like to thank Mr. Allan Nisbet for his assistance in editing the manuscript. This work was supported by a grant from Formation of Innovation Center for Fusion of Advanced Technologies in the Special Coordination Funds for Promoting Science and Technology "Cell Sheet Tissue Engineering Center (CSTEC)" from the Ministry of Education, Culture, Sports Science, and Technology (MEXT), Japan.

\section{References}

1. Walsh G (2014) Biopharmaceutical benchmarks 2014. Nat Biotechnol 32: 992-1000.

2. Wurm FM (2004) Production of recombinant protein therapeutics in cultivated mammalian cells. Nat Biotechnol 22: 1393-1398.

3. Chaudhary S, Pak JE, Gruswitz F, Sharma V, Stroud RM (2012) Overexpressing human membrane proteins in stably transfected and clonal human embryonic kidney 293S cells. Nat Protoc 7: 453-466.

4. Loignon M, Perret S, Kelly J, Boulais D, Cass B, et al. (2008) Stable high volumetric production of glycosylated human recombinant IFNalpha2b in HEK293 cells. BMC Biotechnol 8: 65.

5. Parham J, Kost T, Hutchins J (2001) Effects of pCIneo and pCEP4 expression vectors on transient and stable protein production in human and simian cell lines. Cytotechnology 35: 181-187.

6. Pham PL, Perret S, Doan HC, Cass B, St-Laurent G, et al. (2003) Largescale transient transfection of serum-free suspension-growing HEK293 EBNA1 cells: peptone additives improve cell growth and transfection efficiency. Biotechnol Bioeng 84: 332-342.

7. Jordan M, Köhne C, Wurm FM (1998) Calcium-phosphate mediated DNA transfer into HEK-293 cells in suspension: control of physicochemical parameters allows transfection in stirred media. Transfection and protein expression in mammalian cells. Cytotechnology 26: 39-47.

8. Ryu JH, Kim S, Cho S, Choi CY, Kim B (2004) HEK 293 cell suspension culture using fibronectin-adsorbed polymer nano spheres in serum-free medium. Wiley Periodicals 2004: 128-133.

9. Zweigerdt R, Olmer R, Singh H, Haverich A, Martin U (2011) Scalable expansion of human pluripotent stem cells in suspension culture. Nat Protoc 6: 689-700.

10. Kelley BD (2001) Biochemical engineering: Bioprocessing of therapeutic proteins. Curr Opin Biotechnol 12: 173-174.

11. Haraguchi Y, Ohtsuki A, Oka T, Shimizu T (2015) Electrophysiological analysis of mammalian cells expressing hERG using automated 384-wellpatch-clamp. BMC Pharmacol Toxicol 16: 39.

12. Imming P, Sinning C, Meyer A (2006) Drugs, their targets and the nature and number of drug targets. Nat Rev Drug Discov 5: 821-834.

13. Durocher Y, Perret S, Kamen A (2002) High-level and high-throughput recombinant protein production by transient transfection of suspensiongrowing human 293-EBNA1 cells. Nucleic Acids Res 30: E9. 
Citation: Mohd Zin NK, Sakaguchi K, Haraguchi Y, Takahashi A, Suzuki S, et al. (2016) Controlling Shear Stress in a Suspension Culture using Couette Flow for Efficient Proliferation of HEK 293 Cells. Fluid Mech Open Acc 3: 124. doi:10.4172/2476-2296.1000124

Page 5 of 5

14. Jakovljevic M, Milovanovic O (2012) Pharmacological in vitro models in pre-clinical drug testing - example of hSERT transfected human embryonic kidney cells. Acts Medica 51: 34-38.

15. Thomson JA, Itskovitz-Eldor J, Shapiro SS, Waknitz MA, Swiergiel JJ, et al. (1998) Embryonic stem cell lines derived from human blastocysts. Science 282: 1145-1147.

16. Takahashi K, Tanabe K, Ohnuki M, Narita M, Ichisaka T, et al. (2007) Induction of pluripotent stem cells from adult human fibroblasts by defined factors. Cell 131: 861-872.

17. Yu J, Vodyanik MA, Smuga-Otto K, Antosiewicz-Bourget J, Frane JL, et al. (2007) Induced pluripotent stem cell lines derived from human somatic cells. Science 318: 1917-1920.

18. Laustriat D, Gide J, Peschanski M (2010) Human pluripotent stem cells in drug discovery and predictive toxicology. Biochem Soc Trans 38: 1051-1057.

19. Priori SG, Napolitano C, Di Pasquale E, Condorelli G (2013) Induced pluripotent stem cell-derived cardiomyocytes in studies of inherited arrhythmias. J Clin Invest 123: 84-91.

20. Singh H, Mok P, Balakrishnan T, Rahmat SN, Zweigerdt R (2010) Upscaling single cell-inoculated suspension culture of human embryonic stem cells. Stem Cell Res 4: 165-179.

21. Amit M, Laevsky I, Miropolsky Y, Shariki K, Peri M, et al. (2011) Dynamic suspension culture for scalable expansion of undifferentiated human pluripotent stem cells. Nat Protoc 6: 572-579.

22. Matsuura K, Wada M, Shimizu T, Haraguchi Y, Sato F, et al. (2012) Creation of human cardiac cell sheets using pluripotent stem cells. Biochem Biophys Res Commun 425: 321-327.

23. Chen VC, Couture SM, Ye J, Lin Z, Hua G, et al. (2012) Scalable GMP compliant suspension culture system for human ES cells. Stem Cell Res 8: 388-402.

24. Haraguchi Y, Matsuura K, Shimizu T, Yamato M, Okano T (2015) Simple suspension culture system of human iPS cells maintaining their pluripotency for cardiac cell sheet engineering. J Tissue Eng Regen Med 9: 1363-1375.

25. Kelly WJ (2008) Using computational fluid dynamics to characterize and improve bioreactor performance. Biotechnol Appl Biochem 49: 225-238.

26. Wendl MC (1999) General solution for the Couette flow profile. Phys Rev E Stat Phys Plasmas Fluids Relat Interdiscip Topics 60: 6192-6194.

27. Munson BR, Young DF, Okiishi TH (2012) Fundamentals of Fluid Mechanics (7th Edn.) John Wiley and Sons, India.

28. Yagi T, Shinke M, Takahashi S, Tobe Y, Umezu M, et al. (2011) Experimental insight into spatial and temporal dynamics of wall shear rate using elastic replicas of human cerebral aneurysms with fluorescent stereo PIV, The 11th Asian Symposium on Visualization (ASV 11) Program and Abstracts, 98.

29. Haraguchi Y, Sekine W, Shimizu T, Yamato M, Miyoshi S, et al. (2010) Development of a new assay system for evaluating the permeability of various substances through three-dimensional tissue. Tissue Eng Part C Methods 16: 685-692.

30. Sekine W, Haraguchi Y, Shimizu T, Umezawa A, Okano T (2011) Thickness limitation and cell viability of multi-layered cell sheets and overcoming the diffusion limit by a porous-membrane culture insert. J Biochip Tissue chip S2: 001.

31. Tadakuma K, Tanaka N, Haraguchi Y, Higashimori M, Kaneko M, et al. (2013) Development of a simple device for transfer/transplantation of living cell sheets rapidly and completely without cell damage. Biomaterials 34: 9018-9025.

32. Hasegawa A, Haraguchi Y, Shimizu T, Okano T (2015) Rapid fabrication system for three-dimensional tissues using cell sheet engineering and centrifugation. J Biomed Mater Res A 103: 3825-3833.

33. Zhang Y, Stobbe P, Silvander CO, Chotteau V (2015) Very high cell density perfusion of $\mathrm{CHO}$ cells anchored in a non-woven matrix-based bioreactor. J Biotechnol 213: 28-41. 